\title{
Electronic Structure, Stark Effect and Optical Properties of Multistacked Dots
}

\author{
Angela VASAnelli ${ }^{1,2}$, Milena De GIORGi ${ }^{3}$, Robson FerReira ${ }^{1}$, Roberto Cingolani ${ }^{3}$, \\ Hiroyuki SAKAKI ${ }^{4}$ and Gérald BASTARD ${ }^{1}$ \\ ${ }^{1}$ Laboratoire de Physique ENS, 24 rue Lhomond F-75005 Paris, France \\ ${ }^{2}$ CNR-Centro di Studio Chimica dei Plasmi, via Orabona 4, I-70126 Bari, Italy \\ ${ }^{3}$ INFM, Università di Lecce, via per Arnesano, I-73100 Lecce, Italy \\ ${ }^{4}$ IIS, University of Tokyo, 5-7-10 Roppongi, Minato-ku, Tokyo 106-8558, Japan \\ (Received August 21, 2000; accepted for publication December 19, 2000)
}

We present the results of effective mass calculations for the electronic structure and optical properties of stacked quantum dots in the presence of a longitudinal electric field. Attention is focussed on strongly stacked dot structures, in which a vertical field acts on the electron in the same way as found in a quantum well. We have studied the dipole selection rules and oscillator strengths of stacked dots for intraband transitions (far-infrared absorption). In the case of interband transitions, we have studied the effect of a longitudinal electric field on the overlap between electron and hole wavefunctions. We have also calculated the absorption spectrum for structures of attached dots, taking into account Gaussian fluctuations in the vertical and lateral dimensions.

KEYWORDS: stacked dots, Stark effect, Gaussian fluctuations

Self-assembled semiconductor quantum dots such as InAs are of considerable interest both from the physical and technological points of view, due to their nanometric size and the zero-dimensional nature of their electronic states. One way to achieve large dot concentrations is to stack the quantum dots vertically by taking advantage of the fact that the strain field favors nucleation sites on top of already grown dots. State-ofthe-art growth techniques enable one to obtain a wide variety of structures, ranging from multistacked dots (very weakly interacting with each other) to strongly stacked ones, when there is no GaAs barrier between the dots. We are particularly interested in the latter kind of structure, which has recently been used to manufacture lasing devices. ${ }^{1)}$ It is very different from the structures studied by Pryor ${ }^{2)}$ (very weakly interacting quantum dots) and those experimentally studied by Fry et al. ${ }^{3)}$ (single quantum dots).

In Fig. 1 we show a structure of five attached dots. A single dot is approximated by a truncated cone floating on a onemonolayer-thick wetting layer. We assume the structure displays cylindrical symmetry, ${ }^{4}$ which is a reasonable assumption, as one can infer from the magneto-optical data on single quantum dots. ${ }^{5)}$ The structures we are interested in contain a large number of elementary cells and therefore can be rightfully studied in the frame of an effective mass calculation. The one electron (hole) Schrödinger equation is

$$
H_{\mathrm{dot}} \Psi(\mathbf{r})=\left(\frac{\mathbf{p}^{2}}{2 m^{*}}+V_{\mathrm{dot}}(\mathbf{r})\right) \Psi(\mathbf{r})=E \Psi(\mathbf{r}),
$$

where $V_{\text {dot }}(\mathbf{r})$ is an axially symmetric confining potential for electrons (holes) in the dot, which is zero in the GaAs barrier and equal to $V_{\mathrm{e}}=-413 \mathrm{meV}\left(V_{\mathrm{h}}=-288 \mathrm{meV}\right)$ in the dot. The effective mass for electrons is taken as equal to 0.067 $m_{0}$, while for the holes, we take an anisotropic effective mass, $0.112 m_{0}$ in the plane and $0.337 m_{0}$ along the stacking direction. Because of the cylindrical symmetry, the solutions may be written as

$$
\Psi_{\mathrm{nl}}(\mathbf{r})=\frac{e^{i l \vartheta}}{\sqrt{2 \pi}} \psi_{\mathrm{nl}}(\rho, z),
$$

with $l=0$ for S-like levels, $l= \pm 1$ for P-like levels and so

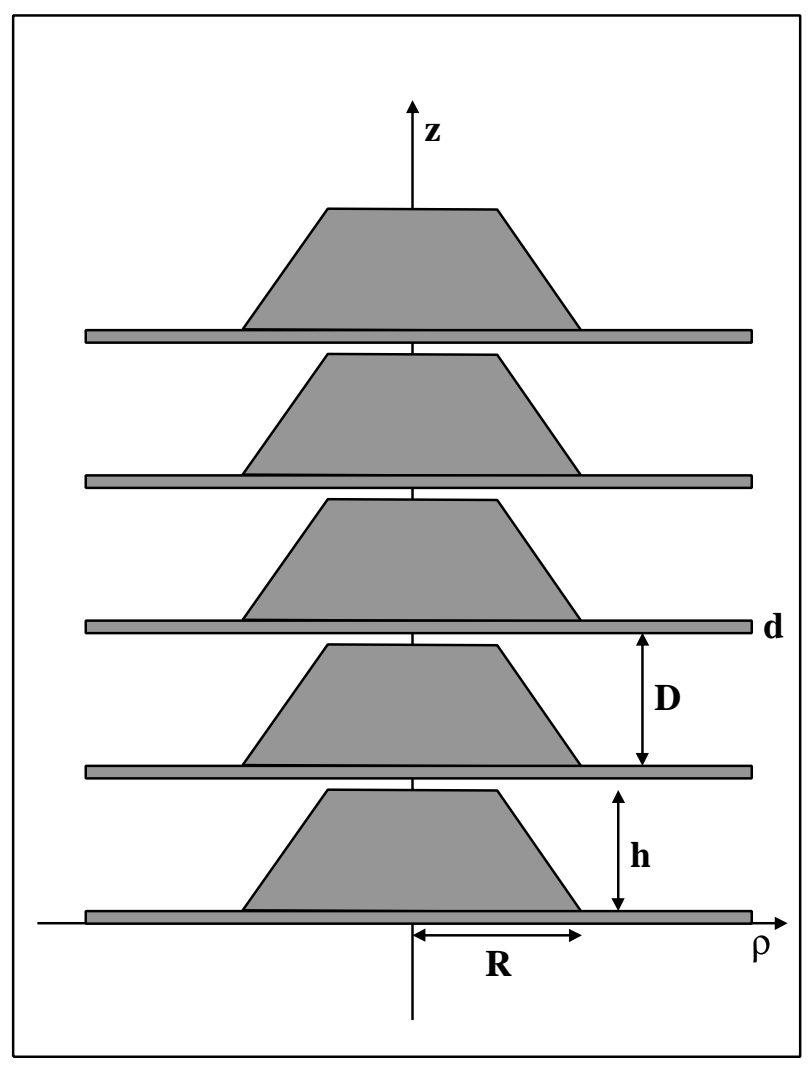

Fig. 1. Structure of five dots. Zones in grey are the InAs regions.

forth. For bound levels we consider the separable form

$$
\begin{aligned}
\psi_{\mathrm{nl}}(\rho, z) & =F_{\mathrm{nl}}(\rho) \varphi_{\mathrm{nl}}(z) \\
F_{\mathrm{nl}}(\rho) & =N_{\mathrm{nl}} \rho^{|l|} P_{\mathrm{nl}}\left(\rho^{2}\right) \exp \left(-\frac{\rho^{2}}{2 \beta_{\mathrm{nl}}^{2}}\right) \\
P_{\mathrm{nl}}\left(\rho^{2}\right) & =\sum_{p=0}^{n-1} a_{\mathrm{p}, \mathrm{nl}} \rho^{2 p},
\end{aligned}
$$

where $N_{\mathrm{nl}}$ are normalization constants and $a_{0, \mathrm{nl}}=1$. The coefficients of the polynomial are obtained by imposing the orthonormalization conditions between the wavefunctions. By 


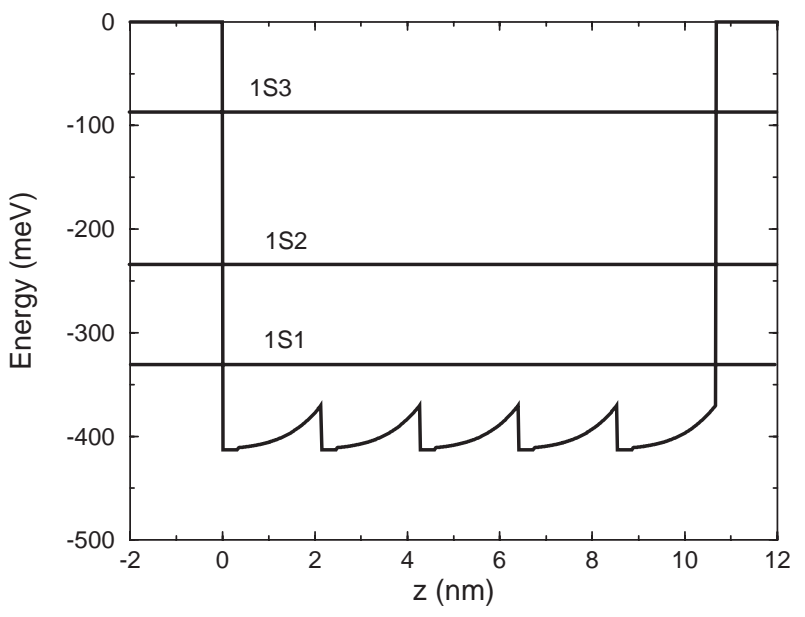

Fig. 2. Effective potential of an electron in the $z$ direction for the $1 S$-like states for a structure of five attached dots $(R=90 \AA, h=18 \AA$, basis angle $\left.=30^{\circ}\right)$. The horizontal bars give the calculated energies of the three $1 S p$ bound states $(p=1,2,3)$.

substituting the expression of the wavefunctions in eq. (1), we obtain a 1D Schrödinger equation, with an effective potential in the $z$ direction. Figure 2 shows the calculated effective potential felt by an electron along the $z$ direction of a structure of five attached dots. We see that the potential modulation along the $z$ direction is very weak and that, to a first approximation, the electron moves rather freely along the vertical corridor. To be more quantitative, let us consider a structure of seven attached quantum dots with dimensions $R_{0}=90 \AA, h_{0}=18 \AA$ and $\alpha=30^{\circ} \mathrm{C}$. We calculate four bound $1 S$ levels in this structure: $E_{1 S 1}=-344.7 \mathrm{meV}, E_{1 S 2}=-289.3 \mathrm{meV}, E_{1 S 3}=$ $-199 \mathrm{meV}, E_{1 S 4}=-79.2 \mathrm{meV}$, while the parameter for the in-plane motion is $\beta_{1 S}=39 \AA$. This corresponds to a transverse kinetic energy of $\left\langle T_{\perp}\right\rangle_{1 S} \simeq 37.3 \mathrm{meV}$. For a square well with $150 \AA$ thickness (which is equal to the total length of the multidot structure, i.e. $7 \times(3.33+18) \AA$ ) with a potential depth of $-413 \mathrm{meV}$, we also find four bound states. If one adds the transverse kinetic energy to the quantum-well eigenstates to artificially reproduce the multidot situation, we find the energies of the four quantum well bound states $E_{1 \rightarrow 4}$ equal to $E_{1}+\left\langle T_{\perp}\right\rangle_{1 S} \simeq-356 \mathrm{meV}, E_{2}+\left\langle T_{\perp}\right\rangle_{1 S} \simeq-301.2 \mathrm{meV}$, $E_{3}+\left\langle T_{\perp}\right\rangle_{1 S} \simeq-210.4 \mathrm{meV}, E_{4}+\left\langle T_{\perp}\right\rangle_{1 S} \simeq-89.5 \mathrm{meV}$. There is therefore a very good agreement between both calculations, the difference (an upward shift of $\simeq 11 \mathrm{meV}$ ) being almost entirely accounted for by the average value of the potential modulation along $z$. Hence, for tightly stacked dots the picture of a cylinderlike 3D profile gives a good estimate of the $1 S p$ electron eigenstates. This is essentially due to the fact that the dots touch each other and the InAs region forms a corridor inside the GaAs barrier, with an effective radial thickness controlled by $\beta_{1 S}$. Note that the cylindric potential approximation becomes less valid for $l \neq 0$ states, because in this case the departures of the effective potential from the quantum-well potential are more important.

This behavior, which is reminiscent of the pseudoalloy regime in the case of 1D strongly coupled superlattices, seems very interesting because it enables the creation of artificial quantum wells while at the same time avoids the formation of a $2 \mathrm{D}$ continuum like the one found in the case of a regular quantum well.

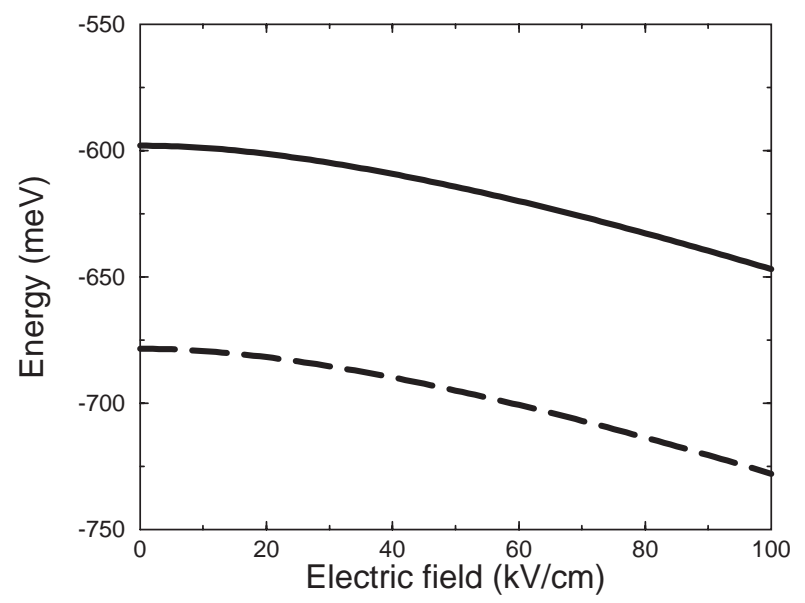

Fig. 3. Fundamental transition energy (origin at the GaAs gap) versus electric field in a structure of seven attached dots (solid line), and in a quantum well (dashed line) with the same vertical dimension $(L=150 \AA$ $=7 \times(3.33+18) \AA$ ).

Next, we study the effect of a uniform electric field

$$
H=H_{\mathrm{dot}} \pm e F z
$$

for electrons $(+)$ and holes $(-)$. We write the total wavefunction as a linear combination of all unperturbed bound states. Thus, the electric perturbation is exactly diagonalized within this truncated basis and the eigenenergies in the presence of $\mathrm{F}$ are obtained by solving

$$
\sum_{\mathrm{nlp}}\left(\left\langle\psi_{\mathrm{n}^{\prime} \mathrm{l}^{\prime} \mathrm{p}^{\prime}}|H| \psi_{\mathrm{nlp}}\right\rangle-E\left\langle\psi_{\mathrm{n}^{\prime} l^{\prime} \mathrm{p}^{\prime}} \mid \psi_{\mathrm{nlp}}\right\rangle\right) c_{n l p}=0
$$

Note that, due to the orthogonalization relations between the unperturbed wavefunctions, the electric field has an effect on the ground state only if there are $1 S p$ excited states in the multidot structure. For quantum dots with $R_{0}, h_{0}$, and $\alpha$, an excited $1 S$ state starts to be bound for a structure of three attached dots. In Fig. 3, using a solid line, we indicate the energy of the fundamental electron-hole transition (the origin of the energy is at the GaAs gap) with respect to the electric field. This is compared to the fundamental transition in a quantum well with the same dimensions (dashed line). It is evident that the electric field acts on electrons and holes in the two structures in the same way and, as at zero field, the blue shift of the dot structure with respect to the quantum well is mostly due to the radial confinement. Hence, even in the presence of an electric field, strongly coupled dots and quantum-well structures $^{6)}$ display the same electronic behavior for the $z$ motion; on the other hand, the transverse electron motion is confined for dot structures, while it is free for quantum wells. The suppression of the $2 \mathrm{D}$ continuum may be a significant advantage for electro-optical applications.

Once the transition energies for a given electric field are known, the absorption spectrum should be the sum of delta functions weighted by the squared overlaps between the electron and hole envelopes. Actually, quantum dots exhibit size fluctuations around an average value. Hence, the absorption spectrum of an ensemble of stacks is a broad line, whose width depends on the size fluctuations through the variation of the electron and hole energies with respect to $R$ and $h=\sum_{\mathrm{i}} h_{\mathrm{i}}$, where $i$ labels the dots of a given stack. Let us assume that $R$ and $h=\sum_{\mathrm{i}} h_{\mathrm{i}}$ distributions are Gaussian, cen- 


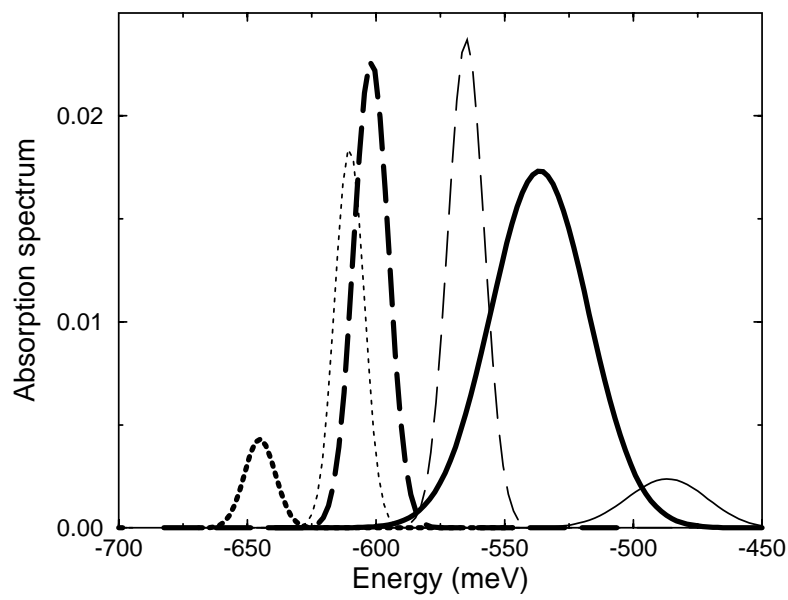

Fig. 4. Absorption spectra for the fundamental (thick lines) and for the first excited transition (thin lines) for a structure of three (solid lines), five (dashed lines) and seven (dotted lines) dots. The structures are subjected to a $100 \mathrm{kV} / \mathrm{cm}$ electric field.

tered, respectively, at $R_{0}$ and $h_{0}$ with standard deviations of $\sigma_{\mathrm{R}}=3 \AA$ for the radius and $\sigma_{\mathrm{h}}=6 \AA$ for the height. We assume that a given $h$ fluctuation is equally shared between all dots of a given stack and that the $h$ and $R$ fluctuations are uncorrelated. The absorption probability is easily calculated because the transition energies and overlap integrals vary linearly with $R$ and $h$ for the relevant $R$ and $h$ variations. Hence the absorption probability becomes proportional to $A(\varepsilon)$ where

$$
\begin{aligned}
A(\varepsilon) & =\frac{1}{\sqrt{2 \pi}} \frac{1}{\sigma_{\mathrm{T}}}\left[O v\left(R_{0}, h_{0}\right)-\varepsilon \cdot k\right] \exp \left(-\frac{\varepsilon^{2}}{2 \sigma_{\mathrm{T}}^{2}}\right) \\
k & =\frac{\partial O v}{\partial R} \frac{\sigma_{\mathrm{R}}^{2}}{\sigma_{\mathrm{T}}^{2}}\left|\frac{\partial \varepsilon_{\mathrm{T}}}{\partial R}\right|+\frac{\partial O v}{\partial h} \frac{\sigma_{\mathrm{h}}^{2}}{\sigma_{\mathrm{T}}^{2}}\left|\frac{\partial \varepsilon_{\mathrm{T}}}{\partial h}\right|,
\end{aligned}
$$

with $\varepsilon_{\mathrm{T}}$ the total energy, $O v$ the overlap integral between electron and hole wavefunctions, and $\varepsilon$ the detuning with respect to the most probable transition energy; $\sigma_{\mathrm{T}}$ is the width of the absorption spectrum, given by

$$
\sigma_{\mathrm{T}}^{2}=\sigma_{\mathrm{h}}^{2}\left|\frac{\partial \varepsilon_{\mathrm{T}}}{\partial h}\right|^{2}+\sigma_{\mathrm{R}}^{2}\left|\frac{\partial \varepsilon_{\mathrm{T}}}{\partial R}\right|^{2} .
$$

Note that in eq. (9), $k$ is very small because the overlap integral changes negligibly with the dimensions of the dot, so the expression in brackets does not vanish. Thus, the intensity of the absorption depends mainly on $O v\left(R_{0}, h_{0}\right)$ and on $\sigma_{\mathrm{T}}$. In Fig. 4 we show the absorption spectrum for the fundamental (thick lines) and for the first excited transition (thin lines), for three (solid lines), five (dashed lines) and seven (dotted lines) dot structures in the presence of a $100 \mathrm{kV} / \mathrm{cm}$ electric field. The broadening of the fundamental transition decreases as the number of dots increases. This happens because the derivative of the energy with respect to the height decreases considerably (in absolute value) as the number of dots is increased, while the total energy changes negligibly with the radius in the three cases. This decrease is more pronounced when going from three to five dots $\left(\partial \varepsilon_{\mathrm{T}} / \partial h=-3.05\right.$ for three dots, $\partial \varepsilon_{\mathrm{T}} / \partial h=-0.68$ for five dots, $\partial \varepsilon_{\mathrm{T}} / \partial h=-0.48$ for seven dots), which leads to a larger decrease in the absorption width. The overlap integral for the ground transition decreases as the number of dots increases, since the electric field can separate the electrons and holes more effectively. We calculate the overlap integrals of 0.83 (three dots), 0.38 (five dots) and 0.06 (seven dots). The reason why the absorption peak is more intense in the five dots case than in the three dots case, in spite of the decreasing overlap values, is that the decrease in the $\sigma_{\mathrm{T}}$ value (which is in the denominator of eq. (9) overshadows the decrease of the overlap.

While decreasing the overlap integral for the fundamental state, the electric field makes transitions between electronhole excited states possible, which, in the unperturbed case, are forbidden in practice. From Fig. 4, it is evident that the first excited transition (1S1(e)-1S2(hh)) is more intense than the fundamental one in the five and seven dots cases, where a larger electric-field-induced electron-hole separation takes place. However, the fundamental and excited spectra remain separated by more than the Gaussian width, which may be an advantage for electromodulation.

\section{Acknowledgements}

The LPMC-ENS is "Unité Associée au CNRS (UMR 8551) et aux Universités Paris 6 et Paris 7". This work has been partly supported by a New Energy and Industrial Technology Development Organization (NEDO) grant and by a E.E.C. project (IST-1999-11311 (SQID)).

1) M. Sugarawa, K. Mukai and Y. Nakata: Appl. Phys. Lett. 74 (1999) 1561.

2) C. Pryor: Phys. Rev. Lett. 80 (1998) 3579.

3) P. W. Fry et al.: Phys. Rev. Lett. 84 (2000) 733.

4) A. Vasanelli, M. De Giorgi, R. Ferreira, R. Cingolani and G. Bastard: 2000, unpublished.

5) S. Hameau, Y. Guldner, O. Verzelen, R. Ferreira, G. Bastard, J. Zeman, A. Lemaître and J. M. Gérard: Phys. Rev. Lett. 83 (1999) 4152.

6) G. Bastard, J. A. Brum and R. Ferreira: Solid State Phys. 44 (1991) 229. 